Submission ID: 43814

\title{
Development of Cloud Computing Software for LWD Measurements and
} Geosteering

A.R. Dudaev (NSU, IPGG SB RAS), A.Y. Sobolev (IPGG SB RAS, NSU, NSTU), V.N. Glinskikh* (IPGG SB

RAS, NSU, NSTU)

\section{SUMMARY}

We have developed a prototype of cloud computing software for logging-while-drilling and geosteering. For the first time ever, during the development of software for addressing the problems of petroleum geophysics, we apply software solutions based on state-of-the-art IT-technologies in the field of cloud computing, namely, cross-platform scalable distributed computing. We have created a client-server application written in JavaScript, which stores all user data in MongoDB. The application allows a user to send input data through message queues for processing by means of computing applications that run in a virtual environment. Processing results are displayed on a web page. We have tested the software under discussion on real practical data from the interval of a subhorizontal well from one of the Latitude Priob oil fields. When drilling highly deviated boreholes, an effective well targeting in the productive part of a reservoir is essential. Successful Russian experience of studying oil and gas wells is demonstrated by using the first Russian telemetry system for logging-while-drilling, intended for drilling wells with horizontal completions. At the present time, it is necessary to develop a multifunctional automated computer system for processing, visualization and interpretation of the data obtained with the complex under consideration. 
Программное обеспечение с облачными вычислениями для электромагнитного каротажа в процессе бурения нефтегазовых скважин

\author{
А.Р. Дудаев (НГУ, ИНГГ СО РАН), А.Ю. Соболев (ИНГГ СО РАН, НГУ, НГТУ), \\ В.Н. Глинских* (ИНГГ СО РАН, НГУ, НГТУ)
}

\title{
Введение
}

При наклонно-горизонтальном бурении необходима эффективная проводка ствола скважины в продуктивной части пласта с высокими фильтрационно-ёмкостными свойствами. Изучение горизонтальных скважин традиционно проводится автономными комплексами и во время бурения. Широко используемые комплексы каротажа в процессе бурения разработаны ведущими зарубежными компаниями Baker Hughes, Halliburton, Schlumberger, Weatherford.

Успешный российский опыт изучения нефтегазовых скважин демонстрируется с использованием первой российской телеметрической системы каротажа в процессе бурения, предназначенной для наклонно-направленного бурения скважин с горизонтальным завершением [Еремин и др., 2013; Каюров и др., 2014; Эпов и др., 2014а, 2014б, 2015, 2016а, 2016б; Никитенко и др., 2014, 2015, 2016; Глинских и др., 2017]. Система включает основные геофизические методы исследования в скважинах: инклинометрия, гамма-каротаж, многозондовый высокочастотный индукционный каротаж, нейтрон-нейтронный каротаж, гамма-гамма плотностной каротаж и другие методы.

В настоящее время выполняется разработка многофункциональной автоматизированной компьютерной системы обработки, визуализации и интерпретации данных созданного аппаратурного комплекса каротажа в процессе бурения. Разрабатываемое программное обеспечение (ПО) необходимо для решения задач геонавигации, которая включает определение положения скважины относительно геологических границ и выполняется по данным индукционного каротажа в процессе бурения с оценкой значений удельного электрического сопротивления (УЭС) пластов, положения и наклона их границ.

Стремительное развитие информационных технологий и доступ к высокоскоростной глобальной сети Интернет открывают широкие возможности в различных геофизических приложениях. Применение современных IT-технологий обуславливает принципиально новые подходы к разработке ПО. Использование web-технологий позволяет разрабатывать приложения, интерпретируемые браузерами, незначительно уступающие в производительности, но в более короткой срок по сравнению с написанием на низкоуровневых языках. Этому способствует большое количество интенсивно развивающихся библиотек.

Анализ литературы показывает, что существенных изменений в функциональных возможностях ПО для решения геофизических задач не происходит. Так, известные ПО способны выполнять чтение экспериментальных данных, обработку и интерпретацию, визуализацию результатов и их запись. Отличия же в большей степени состоят в программной реализации. ПО, как правило, разрабатывается в виде единого приложения, требующего установку и выполняющего обработку и визуализацию данных на локальной машине. Среди наиболее известного ПО для решения задач промысловой геофизики, разработанного в ИНГГ СО РАН, отметим МФС ВИКИЗ и ЕМF Рrо [Ельцов и др., 2000; Власов и др., 2008]. Из-за усложнения геофизических задач появляется необходимость в использовании высокопроизводительных вычислителей, а развитие сетевых технологий позволяет производить ресурсоемкие расчеты на удаленных системах [Мартьянов и др., 2011].

Целью настоящей работы является создание ПО на основе современных информационных технологий в области облачных вычислений. Они используют принципиально новые подходы к разработке ПО, основанные на кроссплатформенных распределенных масштабируемых вычислениях. Это позволит работать с каротажными данными и визуализировать результаты их обработки на большинстве устройств от персонального компьютера до мобильного телефона в масштабе реального времени. 


\section{Основные особенности реализации ПО с облачными вычислениями}

Для разработки ПО предлагается использовать облачные вычисления - модель предоставления пользователю масштабируемых вычислительных ресурсов в виде сервиса через глобальную сеть Интернет. Разрабатываемое ПО основано на клиент-серверной архитектуре. Клиентская часть отвечает за визуализацию данных и не требует мощных вычислительных ресурсов. Серверная часть включает выполнение сложных и ресурсоемких задач на высокопроизводительных многопроцессорных серверах (кластерах). Поэтому такое ПО может использоваться на любых устройствах, от мобильных до персональных компьютеров (ПК), на которых имеются браузер и доступ к серверу по сети. За счет использования единого ресурса обновление приложения выполняется для всех клиентов одновременно.

B качестве языка программирования выбран JavaScript, являющийся общераспространенным языком для создания приложений, интерпретируемых интернет-браузерами. Для разработки модулей визуализации данных применяется библиотека D3.js, имеющая требуемые возможности и приемлемые характеристики для создаваемого ПО [D3.js, 2017]. На стороне сервера используется платформа Node.js [Node.js, 2017], позволяющая писать программный код управляющего диспетчера и клиентской части на одном языке.

Для хранения каротажных данных и результатов расчетов авторизированных пользователей используется база данных [MongoDB, 2017]. Выполнение геофизических расчетов начинается с запуска вычислительного модуля и передачи ему исходных данных через систему обмена сообщений (Message-Oriented Middleware, MOM) [RabbitMQ, 2017]. Для запуска модулей используются платформы [Docker, 2017], предварительно развернутые на каждой удаленной машине и в последующем кластеризованные [Docker Swarm, 2017], что дает возможность выполнять вычисления на наиболее свободных ресурсах. Вычислительные программы с необходимыми им зависимостями выполняются в изолированной виртуальной среде. Как необходимые для вычислений входные данные, так и результаты расчетов запрашиваются и возвращаются модулем через МОМ с последующей визуализацией пользователю в браузере.

Используемый подход за счет гибкой архитектуры позволяет легко масштабировать количество вычислительных узлов и программ, а также подключать модули, написанные на низкоуровневых языках, для получения наибольшей производительности. Такой подход предоставляет возможность простого переиспользования готовых модулей помещением в изолированное настраиваемое окружение.

Возможности нового подхода демонстрируются на примере разработки ПО для электромагнитного каротажа в процессе бурения наклонно-горизонтальных скважин применительно к задачам геонавигации. Комплекс для каротажа в процессе бурения [Еремин и др., 2013; Каюров и др., 2014; Эпов и др., 2015; Никитенко и др., 2016; Глинских и др., 2017] позволяет в масштабе реального времени определять характеристики флюидонасыщенных пластов-коллекторов, вскрытых наклонно-горизонтальными скважинами, определять расстояние до кровли и/или подошвы пласта во вмещающих породах и водонефтяного контакта, угол наклона скважины относительно границ пластов, а также их УЭС. Численная инверсия данных электромагнитного каротажа выполняется в рамках дву-, трех- или многослойной горизонтально-слоистой модели среды с учетом наклона зондов относительно границ пластов в заданном интервале (инверсионном окне) скважины. Последовательно полученные результаты численной инверсии данных электромагнитного каротажа, показывающие изменение УЭС пластов и положения их границ вдоль скважины, позволяют проводить стол скважины в продуктивной части коллектора. Применение такого оконного подхода к инверсии данных обуславливает возможность организации параллельных вычислений данных и их оперативной визуализации.

\section{Описание прототипа ПО для каротажа в процессе бурения}

Ниже приведен внешний вид прототипа разрабатываемого ПО с облачными вычислениями для каротажа в процессе бурения скважин и решения задач геонавигации и результаты его тестирования (рис. 1). Демонстрируются результаты отображения практических данных и результатов их обработки из интервала субгоризонтальной скважины одного из 
месторождений Широтного Приобья. Вверху показаны практические записи сигналов многозондового высокочастотного индукционного каротажа в процессе бурения и результат их трансформации в кажущееся УЭС из интервала наклонно-горизонтальной скважины. Внизу слева приведены данные этого же метода из интервала опорной вертикальной скважины, расположенной рядом с изучаемой субгоризонтальной. Показаны диаграммы кажущегося УЭС основных зондов индукционного каротажа и вертикальное распределение УЭС пластов геоэлектрической модели, полученной при их обработке. Внизу справа приведена геоэлектрическая модель, построенная на основе распределения кажущегося УЭС одного из зондов. Положение кровли продуктивного горизонта корректируется по априорным данным структурной карты.
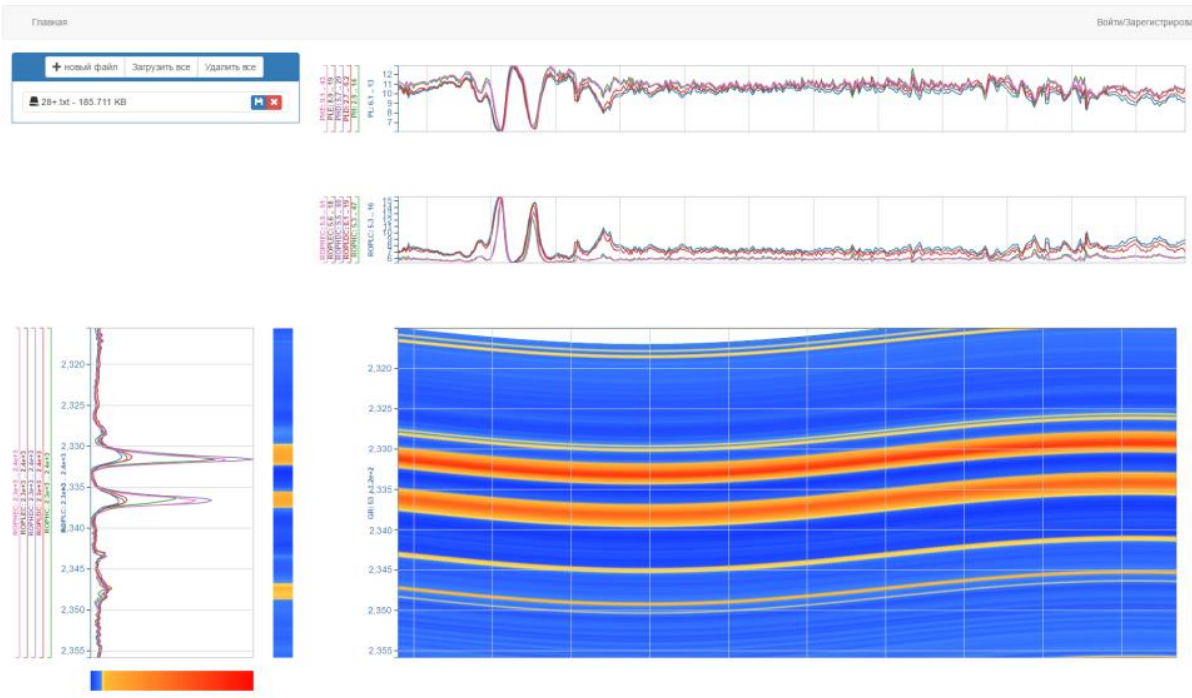

Рисунок 1 Внешний вид прототипа разрабатываемого ПО с облачными вычислениями для каротажа в прочессе бурения скважин и решения задач геонавигаиии.

\section{Выводы}

Таким образом, предложен облачный сервис для обработки и интерпретации геофизических данных с использованием кроссплатформенных распределенных масштабируемых вычислений. Рассмотрены основные особенности реализации ПО, включая применение изолированных виртуальных сред, системы обмена сообщений МОМ, базы данных и управляющего диспетчера на платформе Node.js. Результаты работы представляют значительный интерес для создания системы обработки и интерпретации данных отечественной телеметрической системы каротажа в процессе бурения.

\section{Библиография}

Власов, А.А., Малеева, Л.В., Пудова, М.А., Ельцов, И.Н. [2008] Система комплексной интерпретации каротажных данных EMF PRO. ГЕО-Сибирь-2008.: сб. материалов IV Международного научного конгресса. - Новосибирск: СГГА, с. 132-137.

Глинских, В.Н., Никитенко, М.Н., Эпов, М.И., Еремин, В.Н. [2017] Алгоритмы и программное обеспечение для геонавигации по данным электромагнитного каротажа в процессе бурения. 2-я научно-практическая конференция «Горизонтальные скважины 2017: Проблемы и перспективы», 3G05.

Ельцов, И.Н., Эпов, М.И., Ульянов, В.Н. и др. [2000] Анализ и инверсия каротажных диаграмм в системе МФС ВИКИЗ-98. Каротажник, 73, с. 70-84.

Еремин, В.Н., Волканин, Ю.М., Тарасов, А.В. [2013] Аппаратурно-методическое обеспечение электромагнитного каротажа в процессе бурения. Каротажник, 226, с. 62-69. 
Каюров, К.Н., Еремин, В.Н., Эпов, М.И., Глинских, В.Н., Сухорукова, К.В., Никитенко, М.Н. [2014] Аппаратура и интерпретационная база электромагнитного каротажа в процессе бурения. Нефтяное хозяйство, 12, с. 112-115.

Мартьянов, А.С., Тейтельбаум, Д.В., Сердюк, К.С. и др. [2011] Использование свободных сетевых ресурсов предприятия для решения емких вычислительных геофизических задач. Каротажник, 11, с. 56-64.

Никитенко, М.Н., Сухорукова, К.В., Глинских, В.Н., Еремин, В.Н. [2015] Оперативная обработка и интерпретация данных электромагнитного каротажа на разных этапах строительства нефтегазовой скважины. 4-ая международная научно-практическая конференция «Тюмень-2015»: Глубокие горизонты науки и недр, P06. DOI: 10.3997/22144609.201412086.

Эпов, М.И., Никитенко, М.Н., Сухорукова, К.В., Глинских, В.Н., Еремин, В.Н., Горбатенко, А.А., Павлова, М.А. [2014] Сигналы электромагнитного каротажа в процессе бурения и их численная инверсия. Геология и минерально-сырьевые ресурсы Сибири, 3(19), с. 49-55.

Эпов, М.И., Глинских, В.Н., Сухорукова, К.В., Никитенко, М.Н., Еремин, В.Н. [2015] Численное моделирование и инверсия данных электромагнитного каротажа в процессе бурения и шаблонирования нефтегазовых скважин. Геология и геофизика, 56(8), 1520-1529.

Эпов, М.И., Никитенко, М.Н., Глинских, В.Н., Еремин, В.Н. [2016] Изучение электрической макроанизотропии интервалов наклонно-горизонтальных скважин по данным высокочастотного индукционного каротажа в процессе бурения. Каротажник, 269, с. 94-109.

Эпов, М.И., Никитенко, М.Н., Глинских, В.Н., Сухорукова, К.В. [2014] Численное моделирование и анализ сигналов электромагнитного каротажа в процессе бурения. Каротажник, 245, с. 29-42.

Эпов, М.И., Никитенко, М.Н., Сухорукова, К.В., Глинских, В.Н. [2016] Исследование возможностей электрического и электромагнитного каротажа в электрически макроанизотропных пластах, вскрытых наклонно-горизонтальными скважинами. Каротажник, 260, с. 64-79.

D3.js Documentation [2017] URL: https://github.com/d3/d3/wiki

Docker Documentation [2017] URL: https://www.docker.com

Docker Swarm Documentation [2017] URL: https://docs.docker.com/engine/swarm

MongoDB Documentation [2017] URL: https://docs.mongodb.com

Nikitenko, M.N., Glinskikh, V.N., Eremin, V.N., Epov, M.I. [2016] High-frequency LWD Electromagnetic Measurements Applied to Studying Macroanisotropic Properties of Thin-bedded Sedimentary Deposits. SPE Russian Petroleum Technology Conference and Exhibition, SPE-182088MS.

Nikitenko, M.N., Epov, M.I., Glinskikh, V.N., Suhorukova, K.V., Eremin, V.N. [2014] Development of LWD High-frequency Resistivity Tool. 6th Saint Petersburg International Conference \& Exhibition «Geosciences - Investing in the Future», ThD05. DOI: 10.3997/2214-4609.20140177.

Node.js Documentation [2017] URL: https://nodejs.org

RabbitMQ Documentation [2017] URL: https://www.rabbitmq.com/documentation.html

\section{References}

Vlasov, A.A., Maleeva, L.V., Pudova, M.A., Yeltsov, I.N. [2008] Logging data joint inversion system EMF PRO. GEO-Siberia-2008: materials of the IV International Scientific Congress. - Novosibirsk: SSAG, p. 132-137.

Glinskikh, V.N., Nikitenko, M.N., Epov, M.I., Eremin, V.N. [2017] Algorithms and software for geosteering based on electromagnetic logging while drilling data. 2 nd research-to-practice conference «Horizontal wells 2017: Problems and prospects», 3G05.

Yeltsov, I.N., Epov, M.I., Ulyanov, V.N. [2000] Analysis and inversion of well logs in the MFS VIKIZ-98 system. Karotazhnik, 73, p. 70-84.

Eremin, V.N., Volkanin, Yu.M., Tarasov, A.V. [2013] Hardware and methodological support for electromagnetic logging while drilling. Karotazhnik, 226, p. 62-69. 


\section{EAGE

Kayurov, K.N., Eremin, V.N., Epov, M.I., Glinskikh, V.N., Sukhorukova, K.V., Nikitenko, M.N. [2014] Electromagnetic-logging-while-drilling equipment and numerical inversion software. Oil industry, 12, p. 112-115.

Martyanov, A.S., Teytelbaum, D.V., Serdyuk, K.S. [2011] Using vacant network resources of a company for solving resource-intensive geophysical problems. Karotazhnik, 11, p. 56-64.

Nikitenko, M.N., Sukhorukova, K.V., Glinskikh, V.N., Eremin, V.N. [2015] Real-time processing and interpretation of electromagnetic logging data at different stages of well construction. 4th international research-to-practice conference «Tyumen-2015»: Deep horizons of science and mineral resources, P06. DOI: 10.3997 / 2214-4609.201412086.

Epov, M.I., Nikitenko, M.N., Sukhorukova, K.V., Glinskikh, V.N., Eremin, V.N., Gorbatenko, A.A., Pavlova, M.A. [2014] Electromagnetic LWD signals and their numerical inversion. Geology and mineral resources of Siberia, 3(19), p. 49-55.

Epov, M.I., Glinskikh, V.N., Sukhorukova, K.V., Nikitenko, M.N., Eremin, V.N. [2015] Forward modeling and inversion of LWD induction data. Russian Geology and Geophysics, 2015, 56(8), p. 1194-1200.

Epov, M.I., Nikitenko, M.N., Glinskikh, V.N., Eremin, V.N. [2016] Studying the electric macroanisotropy in deviated and horizontal boreholes according to high-frequency induction logging while drilling data. Karotazhnik, 269, p. 94-109.

Epov, M.I., Nikitenko, M.N., Glinskikh, V.N., Sukhorukova, K.V. [2014] Numerical simulation and analysis of electromagnetic logging-while-drilling signals. Karotazhnik, 245, 29-42.

Epov, M.I., Nikitenko, M.N., Sukhorukova, K.V., Glinskikh, V.N. [2016] Investigation of the possibilities of electric and electromagnetic logging in electrically macroanizotropic formations penetrated by deviated and horizontal boreholes. Karotazhnik, 260, 64-79.

D3.js Documentation [2017] URL: https://github.com/d3/d3/wiki

Docker Documentation [2017] URL: https://www.docker.com

Docker Swarm Documentation [2017] URL: https://docs.docker.com/engine/swarm

MongoDB Documentation [2017] URL: https://docs.mongodb.com

Nikitenko, M.N., Glinskikh, V.N., Eremin, V.N., Epov, M.I. [2016] High-frequency LWD Electromagnetic Measurements Applied to Studying Macroanisotropic Properties of Thin-bedded Sedimentary Deposits. SPE Russian Petroleum Technology Conference and Exhibition, SPE-182088MS.

Nikitenko, M.N., Epov, M.I., Glinskikh, V.N., Suhorukova, K.V., Eremin, V.N. [2014] Development of LWD High-frequency Resistivity Tool. 6th Saint Petersburg International Conference \& Exhibition "Geosciences - Investing in the Future», ThD05. DOI: 10.3997/2214-4609.20140177.

Node.js Documentation [2017] URL: https://nodejs.org

RabbitMQ Documentation [2017] URL: https://www.rabbitmq.com/documentation.html 